\title{
Measurement of residual nucleus cross sections and recoil energies in $p+$ Fe collisions at 300, 500, 750, 1000 and $1500 \mathrm{MeV}$
}

\author{
C. Villagrasa*, A. Boudard*, J.-E. Ducret*, B. Fernandez*, S. Leray*, C. Volant*,
} W. Wlazło*, P. Armbruster ${ }^{\dagger}$, T. Enqvist ${ }^{\dagger}$, F. Hammache ${ }^{\dagger}$, K. Helariutta ${ }^{\dagger}$, B. Jurado ${ }^{\dagger}$, M.V. Ricciardi ${ }^{\dagger}$, K.-H. Schmidt ${ }^{\dagger}$, K. Sümmerer ${ }^{\dagger}$, F. Vivès ${ }^{\dagger}$, O. Yordanov ${ }^{\dagger}$, L. Audouin ${ }^{* *}$, L. Ferran ${ }^{* *}$, F. Rejmund ${ }^{* *}$, C. Stéphan ${ }^{* *}$, L. Tassan-Got**, J. Benlliure ${ }^{\ddagger}$, E. Casarejos ${ }^{\ddagger}$, M. Fernandez ${ }^{\ddagger}$ J. Pereira ${ }^{\ddagger}$, S. Czajkowski ${ }^{\S}$,

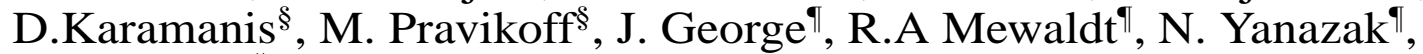
M. Wiedenbeck ${ }^{\|}$, J. Connel ${ }^{\dagger \dagger}$, T. Faestermann ${ }^{\ddagger}$, A. Heinz ${ }^{\S \S}$ and A. Junghans ${ }^{\text {IqI }}$

\author{
*DAPNIA/SPhN CEA/Saclay, F-91191 Gif sur Yvette Cedex, France \\ $\dagger$ GSI, Planckstrasse 1, 64291 Darmstadt, Germany \\ ** IPN Orsay, F-91406 Orsay Cedex, France \\ $\ddagger$ Univ. de Santiago de Compostela, E-15706 Santiago de Compostela, Spain \\ ${ }_{\text {CENBG, } F-33175 \text { Gradignan Cedex, France }}$ \\ ${ }^{\mathbb{}}$ California Institute of Technology, Pasadena, CA 91125 USA \\ ${ }$ Jet Propulsion Laboratory, California Institute of Technology, Pasadena, CA 91109 USA \\ ${ }^{\dagger}$ University of Chicago, Chicago, IL 60637, CA 91125 USA \\ †U Munich, 85747 Garching, Germany \\ ${ }^{\S}$ Argonne National Laboratory, Argonne, IL 60439-4083 USA \\ पIII CENPA/University of Washington, Seattle WA 98195 USA
}

\begin{abstract}
The production of residual nuclei in $\mathrm{p}+\mathrm{Fe}$ collisions has been measured at GSI on the FRS facility by means of the reverse kinematic techniques at 300, 500, 750, 1000 and $1500 \mathrm{MeV} / \mathrm{A}$. The cross-sections larger than $0.01 \mathrm{mb}$ of all isotopes with $\mathrm{Z}$ larger than 8 have been obtained. Velocity distributions were also measured. Comparisons to models describing spallation reactions and some empirical formulae often used in astrophysics are presented. These data are directly used to calculate impurety production and DPAs in a thin window as foreseen in spallation sources or accelerator-driven systems.
\end{abstract}

\section{INTRODUCTION}

The isotopic production of residual nuclei in $\mathrm{p}+\mathrm{Fe}$ collisions has been measured at GSI on the FRS facility by means of the reverse kinematic techniques at 300 , 500, 750, 1000 and $1500 \mathrm{MeV} / \mathrm{A}$ [1]. Cross-sections for all isotopes produced above $0.01 \mathrm{mb}$ with $\mathrm{Z}$ larger than 8 have been obtained. The experiment gives also access to the momentum spectra of each isotope so that, after the proper transformation, the recoil energy of residues in the usual kinematics (proton on iron) can be deduced. These data are interesting in several domains. For astrophysics, they are needed for a detailed knowledge of the spallation process around energies found in cosmic radiations, and for elements of importance in the nucleosynthesis and in the interstellar medium. Compared to previous data [2, 3 ], this experiment provide full isotopic distributions extending to much lower elements on a large range of energies. In spallation neutron sources and acceleratordriven sub-critical reactors, the proton beam accelerated under vacuum have to cross a window before entering the spallation target and the subcritical reactor. In most of the designs, this thin window is dominantly made of iron. The data described here can be directly used to calculate the amount of impurities accumulated and the damage due to the recoil of residual nuclei in such a window. Last, but not least, these iron data are important as a test of spallation models and complement a wide program on isotopic distribution measurements mostly devoted, up to now, to heavy nuclei ( $\mathrm{U}, \mathrm{Pb}$ and $\mathrm{Au})$ [4]. The study of the 
beam energy dependence is also made possible for the first time in this type of experiments.

\section{PRODUCTION CROSS-SECTIONS}

The experiment used the reverse-kinematics method, i.e. a beam of ${ }^{56} \mathrm{Fe}$ onto a liquid hydrogen target. All the fragments produced in the target were therefore strongly forward focused and identified in flight by the fragment separator FRS. The nuclear charge was determined using a multiple sampling ionization chamber. The mass was obtained from the measurements of the magnetic rigidity, velocity and charge of the fragments. The magnetic rigidity of the fragments is determined from the positions at the intermediate and final focal planes measured with plastic scintillators and the knowledge of the ion-optical conditions of the spectrometer. The velocity was experimentally measured by time of flight between the two focal planes (about $36 \mathrm{~m}$ of ToF base). Because of the FRS momentum acceptance $( \pm 1.5 \%)$, several measurements with various magnetic fields were done to cover all the momentum range of the fragments.

The primary-beam intensity was measured from the current induced by secondary electrons in titanium foils. Since the liquid hydrogen target was enclosed between thin titanium foils, measurements were repeated using an identical empty target to allow subtraction of contributions due to the titanium windows.

To obtain the final isotopic production cross-sections, several correction factors were applied which take into account the detection efficiency of the different detctors, the secondary reactions in the different layers of matter in the beam line, the contribution to the reaction rate from multiple reactions inside the hydrogen target, and the FRS transmission correction. The latter is due to the limited geometrical acceptance and ion-optical conditions of the FRS which result in a selection in the angular distribution of the fragments.

Finally, isotopic cross-sections were obtained for elements down to $\mathrm{Z}=10$ at 300 and $500 \mathrm{MeV} / \mathrm{A}$ and $\mathrm{Z}=8$ at $750,1000,1500 \mathrm{MeV} / \mathrm{A}$ with uncertainties around $10 \%$ for most of the fragments [1]. For the lightest ones, the uncertainty could reach $20 \%$ due to the larger applied transmission correction. A comparison with $\gamma$ spectrometry data [5] and with the few previous reverse kinematics data obtained (for a range of charges smaller than ours) by Webber et al. [3] was performed in [1] and showed a reasonable agreement. The charge distribution for the five energies and a selected set of isotopic distributions at $1000 \mathrm{MeV} / \mathrm{A}$ are presented in fig. 1

\section{Comparison with models}

Spallation reactions are generally described by a twostep mechanism: a first stage of individual nucleonnucleon collisions, usually treated by an intranuclear cascade model (INC), then, the decay of the excited remnant nucleus by evaporation-fission. A pre-equilibrium stage is sometimes inserted in-between. Recently, an important effort has been devoted, within the European project HINDAS [6], to the improvement, test and validation on a large set of experimental data of these models. The INCL4 model [7] coupled to the ABLA [8] de-excitation model has been found to satisfactorily reproduce a large number of various observables in different experiments.
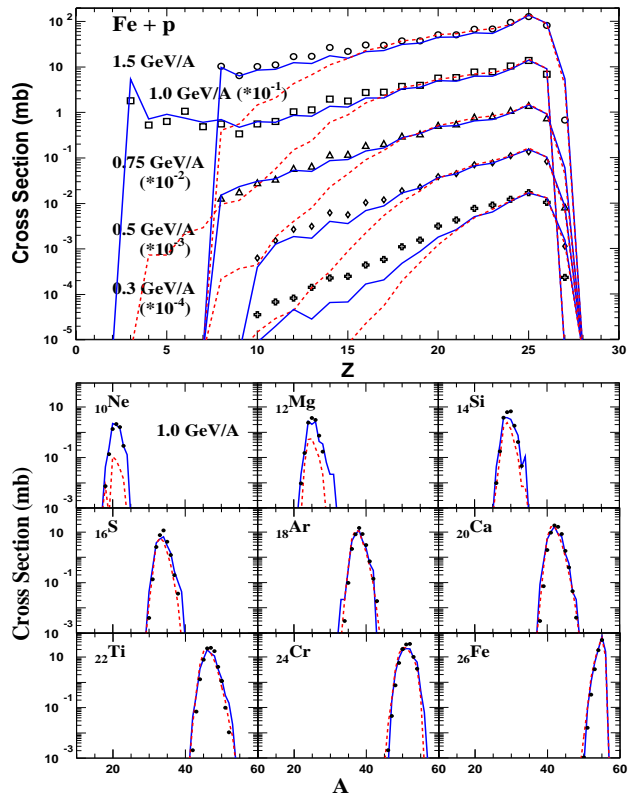

FIGURE 1. Charge distributions at 5 energies (top), including the low charges from [9] at $1 \mathrm{GeV}$. Selected isotopic distributions (bottom). The curves are the predictions of the INCL4 model from [7] with either the de-excitation code ABLA [8] (dashed red line) or GEMINI from [10] (solid blue line).

However, charge and mass distributions measured in $\mathrm{p}+\mathrm{Fe}$ reactions are one of the failures of the model as can be seen in fig. 1. In particular, the decrease of the crosssections with decreasing charges was found too steep compared to the experimental data. Meanwhile, the independent analysis of the very light fragments produced in our experiment at $1 \mathrm{GeV}$ [9], showed that intermediate mass fragments (IMF) $(3 \leq \mathrm{Z} \leq 8)$ were produced in a rather large quantity. In fig. 1 , we also show a comparison of the experimental data with the same INCL4 intranuclear cascade model but followed by the GEMINI de-excitation code from Charity et al. [10]. In the latter model, IMF production is treated as a very asymmetrical fission mode competing with classical evaporation. It 
can be seen that the calculation reproduces quite well the whole charge distributions, including the small even-odd effects observed in the data and the IMF production, except maybe at $300 \mathrm{MeV}$. Also the isotopic distributions, shown for a selected set of elements at $1 \mathrm{GeV}$, are rather well predicted. A similar agreement of GEMINI following another INC model has been recently reported by Mashnik [11]. It has to be noticed however, that the same GEMINI model is not able to reproduce data on heavy nuclei. A way to improve the prediction on light nuclei without loosing the agreement on heavy ones could be to take into account this asymmetrical fission mode of light nuclei into de-excitation models like ABLA.

\section{Comparison to empirical formula}

In astrophysics, spallation residue production crosssections are often derived from empirical formula in which parameters are fitted on experimental data. In fig. 2 we present the comparison of our mass distributions with two of these formulae, from Webber et al. [12] and Silberberg et al. [13]. It can be observed that the Webber formula correctly predicts the highest mass cross-sections while the disagreement increases as the mass decreases. This is probably due to the fact that the parameters were fitted on the available data at that time which were containing only cross-sections for high masses. This illustrates the danger of using parametric formula outside the range on which they were ajusted. On the contrary, the more recent Silberberg and Tsao formula is found to agree reasonably well with our data all along the mass range. This indicates that the mass and the energy dependences in the formula are well controlled.

\section{Recoil velocities}

In addition to the isotopic production cross-sections, a precise value of the longitudinal velocity can be deduced from the magnetic rigidity in the first part of the spectrometer and the mass and charge of the fragments. This allows to obtain velocity distributions for each isotope. Assuming that the reaction takes place in the center of the target, the velocity of the fragment is corrected for the energy loss in the target and transformed into the reference frame of the projectile. Recoil energies can be deduced from a combination of the mean value and the width of the measured velocity dstributions. Fig. 3 shows the mean value of the longitudinal velocity distribution, averaged on isotopes with the same mass, as a function of the mass difference from the projectile. The experimental values are compared to the ones predicted by the INCL4/ABLA combination. A rather large discrepancy
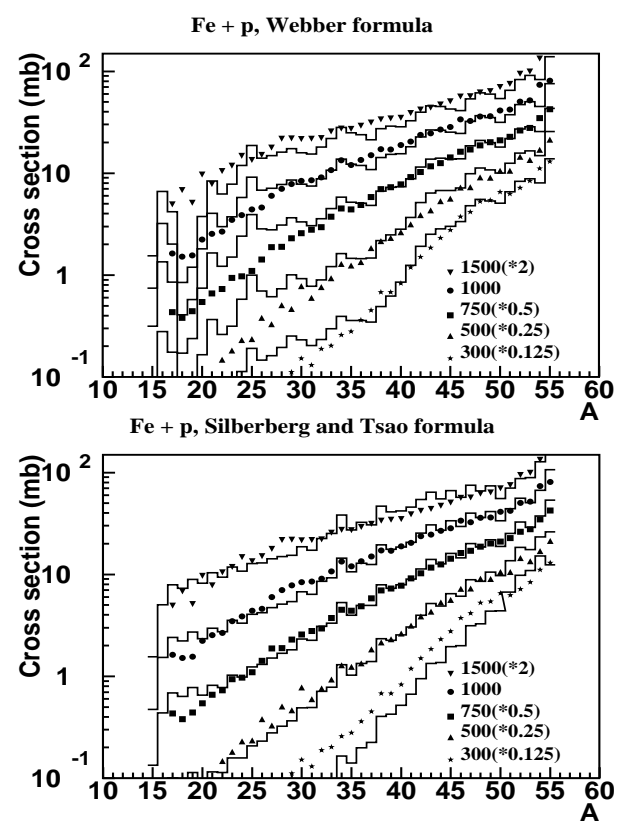

FIGURE 2. Mass distribution at 5 energies compared to Webber formula [12] (top) and to Silberberg and Tsao formula [13] (bottom).

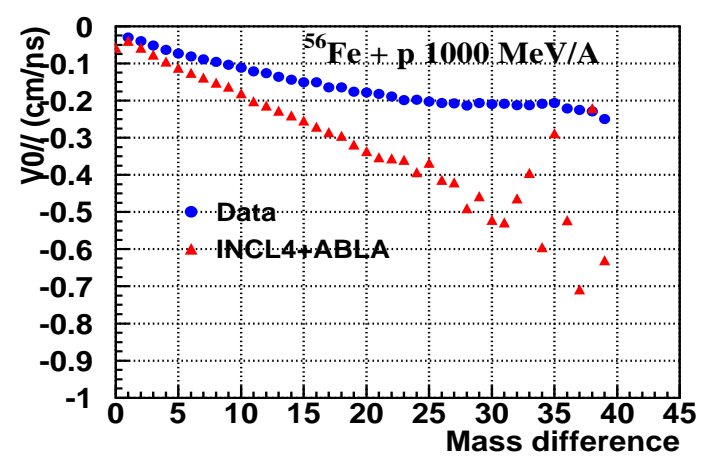

FIGURE 3. Mean value of the longitudinal velocity as a function of the mass difference from the projectile at $1 \mathrm{GeV}$, experimental and calculated with INCL4/ABLA.

is observed: the experimental data do not increase linearly with the mass difference as it is predicted by the theory. Instead, they seem to saturate as the mass difference increases. This behavior is the same for the others energies analyzed in this experiment. This could be related to the fact that low masses could be predominantly produced in asymmetrical fission, as discussed in the preceding section, and therefore have velocity spectrum different from the one predicted by a pure evaporation model. 


\section{APPLICATION TO AN ADS WINDOW}

The window of an ADS can be considered as a thin target and in most of the projects is foreseen to be made of materials containing about $90 \%$ of iron. Therefore, our isotopic cross-sections can be used directly to calculate the chemical impurity produced after a given operation time. Modifications of the chemical composition of the window material possibly result into problems of corrosion or alloy cohesion and modification of mechanical properties. Damages arise also because of atom displacements (DPA) due to residue recoiling. The measured residue longitudinal velocity distributions make it possible to estimate the associated DPAs.

\section{Impurities}

A window of $2 \mathrm{~mm}$ thickness made of iron has been assumed to protect a cylindrical $\mathrm{Pb}-\mathrm{Bi}$ spallation target surrounded by a heavy water moderator. The experimental isotopic cross-section data have used to predict the concentration of the different elements induced by the proton beam, after one year of irradiation with a 32 $\mu \mathrm{A} / \mathrm{cm}^{2}$ proton beam at the five energies. The radioactive decay of the different isotopes during the irradiation time was taken into account. The window is also hit by back-scattered particles coming from the spallation target and the surrounding materials. The LAHET3 code was used to simulate concentration of impurities due to these particles.

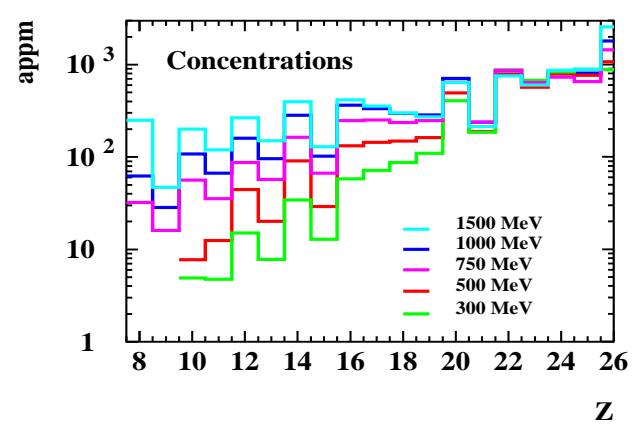

FIGURE 4. Total concentration of impurities at the 5 energies in an iron window after one year of irradiation by a proton beam of $32 \mu \mathrm{A} / \mathrm{cm}^{2}$ of current density.

Final concentrations are given in fig. 4 in appm (atoms of impurities in one million atoms of the window). The most important contribution is due to spallation reactions induced by the direct protons except for iron isotopes. Non-negligible amount of phosphorus, calcium or sulfur, which can be a concern for embrittlement problems in the window material, are found.

\section{Damages due to recoiling residues}

DPAs due to the recoiling residues have been calculated using a method based on the equation of Robinson [14]. Fig.5 shows the results obtained from the experimental cross-sections and recoil energies as a function of the incident energy. Also displayed are the DPAs estimated from the cross-sections and velocities predicted by the Bertini/Dresner $[15,16]$ and INCL4/ABLA. It can be seen that both models give similar results in reasonable agreement with the experimental data, although the energy dependence is slightly different.

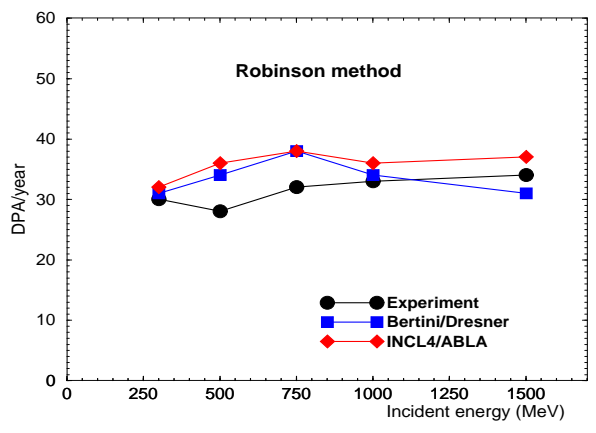

FIGURE 5. Displacements per atom (DPA) versus incident energy in an iron window after one year of irradiation by a $32 \mu \mathrm{A} / \mathrm{cm}^{2}$ proton beam. DPAs are calculated from the experimental cross-sections and recoil velocities and compared to results from Bertini/Dresner and INCL4/ABLA models.

\section{REFERENCES}

1. Villagrasa, C., Report DAPNIA-03-10-7, Ph.D. thesis, Paris XI Orsay (2003).

2. Zeitlin, C., et al., Phys. Rev. C, 56, 388-397 (1997).

3. Webber, W. R., et al., Astrophys. J., 508, 949 (1998).

4. Schmidt, K. H., contribution to this conference (2004).

5. Michel, R., Nucl. Instr. and Meth. in Phys. Res. B, 129, 153-193 (1997).

6. HINDAS, Ec contract fikw-ct-2000-00031, final report (2004).

7. Boudard, A., et al., Phys. Rev. C, 66, 0044615.100446615.28 (2002).

8. Junghans, A. R., et al., Nucl. Phys. A, 629, 635 (1998).

9. Napolitani, P., et al., to appear in Phys. Rev. C (2004).

10. Charity, R. J., Nucl. Phys. A, 483, 391 (1988).

11. Mashnik, S., private communication (2004).

12. Webber, W. R., et al., Phys. Rev. C, 41, 566-571 (1990).

13. Silberberg, R., et al., Astrophys. J., 501, 911-919 (1998).

14. Robinson, M., J. of Nucl. Materials, 216, 1-28 (1994).

15. Bertini, H. W., Phys. Rev., 131, 1801 (1963).

16. Dresner, L., ORNL report, Tech. rep., ORNL-TM-196 (1962). 